\title{
DIMENSION DISTORTION OF IMAGES OF SETS UNDER SOBOLEV MAPPINGS
}

\author{
Stanislav Hencl and Petr Honzík \\ Charles University, Department of Mathematical Analysis \\ Sokolovská 83, 18600 Prague 8, Czech Republic; hencl@karlin.mff.cuni.cz \\ Charles University, Department of Mathematical Analysis \\ Sokolovská 83, 18600 Prague 8, Czech Republic; honzik@gmail.com
}

\begin{abstract}
Let $f: \mathbf{R}^{n} \rightarrow \mathbf{R}^{k}$ be a continuous representative of a mapping in a Sobolev space $W^{1, p}, p>n$. Suppose that the Hausdorff dimension of a set $M$ is at most $\alpha$. Kaufmann [12] proved an optimal bound $\beta=\frac{p \alpha}{p-n+\alpha}$ for the dimension of the image of $M$ under the mapping $f$. We show that this bound remains essentially valid even for $1<p \leq n$ and we also prove analogous bound for mappings in Sobolev spaces with higher order or even fractional smoothness.
\end{abstract}

\section{Introduction}

Let $\Omega \subset \mathbf{R}^{n}$ be an open set and let $f \in W^{s, p}\left(\Omega, \mathbf{R}^{k}\right)$ be a Sobolev mappings. We study the possible dimension of the image $f(M)$ of the set $M \subset \mathbf{R}^{n}$ of Hausdorff dimension $\alpha, 0<\alpha \leq n$. These questions were originally studied in the theory of quasiconformal mappings. Gehring and Väisälä in [8] showed upper and lower bound for the dimension of the image and proved that for every $K$-quasiconformal mapping $f: \Omega \rightarrow \mathbf{R}^{n}$ we have

$$
\operatorname{dim}(f(M)) \leq \frac{p(n, K) \operatorname{dim} M}{p(n, K)-n+\operatorname{dim} M}
$$

where

$$
p(n ; K):=\sup \left\{p: f \in W_{\text {loc }}^{1, p} \text { for every } K \text {-quasiconformal } f \text { in } \mathbf{R}^{n}\right\} .
$$

It was shown by Gehring [7] that $n<p(n, K)$ and the exact value is known only in the plane $p(2, K)=\frac{2 K}{K-1}$ by the result of Astala [2] while it remains open in higher dimension.

It was shown by Kaufman that this upper bound actually holds for every supercritical Sobolev mapping. More precisely, he showed that $\operatorname{dim} f(M) \leq \frac{p \alpha}{p-n+\alpha}$ for every $M \subset \mathbf{R}^{n}$ with $\mathcal{H}^{\alpha}(M)<\infty$ and $f \in W^{1, p}\left(\mathbf{R}^{n}, \mathbf{R}^{k}\right), p>n$. He especially gave a probabilistic construction to demonstrate the sharpness of this result.

Later these results for supercritical Sobolev spaces were extended in the paper Balogh, Monti and Tyson [4] and in the follow up works [3], [5] and [6] that deal also with metric measure spaces. It is well-known that each Sobolev function satisfies the ACL condition, i.e., the function is absolutely continuous when restricted to almost all lines parallel to coordinate axes. It follows that images of $\mathcal{H}^{n-1}$ almost all segments are rectifiable curves and thus have Hausdorff dimension at most one. By

doi:10.5186/aasfm.2015.4026

2010 Mathematics Subject Classification: Primary 46E35, 28A78.

Key words: Sobolev mapping, Hausdorff dimension.

The authors were supported by the ERC CZ grant LL1203 of the Czech Ministry of Education. 
the previously mentioned result of Kaufmann we know that images of $m$-dimensional subspaces have zero $\mathcal{H}^{\frac{p m}{p-n+m}}$ measure. Balogh, Monti and Tyson [4] showed that for any $m<\alpha<\frac{p m}{p-n+m}$ it is true that the image of $\mathcal{H}^{\beta}$ a.e. $m$-dimensional subspace has dimension at most $\alpha$ where $\beta=n-m-\left(1-\frac{m}{\alpha}\right) p$ and by a similar construction as Kaufmann they also showed that this value of $\beta$ is optimal for all $p>n$. These results about the dimension of $\mathcal{H}^{\beta}$-a.e. subspace were extended in the papers of Hencl and Honzík [10] to cover also the subcritical case $p<n$ and later in [11] to cover the case of higher order derivatives or even fractional Sobolev spaces $W^{s, p}$. Here and in the sequal we denote by $W^{s, p}$ the Tribel-Lizorkin spaces $F_{p, 2}^{s}$ which coincide with $W^{k, p}$ for $s=k \in \mathbf{N}$.

The aim of this paper is to return to the paper of Kaufmann and to study similar problem for subcritical Sobolev space $W^{1, p}, p<n$, or even for higher order derivatives and fractional Sobolev spaces $W^{s, p}, s>0$ and $p>1$, both in supercritical $s p>n$ and subcritical case $s p \leq n$. Below we give a full analogy of the result in the supercritical case $s p>n$ for fractional Sobolev spaces which of course includes the result also for higher order Sobolev spaces. Note that this gives us a better result than simply applying Sobolev embedding theorem and using the result of Kaufmann for $s=1$.

Theorem 1.1. Let $n, k \in \mathbf{N}, s>0, p>1, s p>n$ and $0<\alpha \leq n$. Set

$$
\beta=\frac{p \alpha}{s p-n+\alpha}
$$

Suppose that $f \in W^{s, p}\left(\mathbf{R}^{n}, \mathbf{R}^{k}\right)$ is a continuous representative and $M \subset \mathbf{R}^{n}$ is a set with $\operatorname{dim}(M) \leq \alpha$. Then $\operatorname{dim}(f(M)) \leq \max \{\alpha, \beta\}$.

When dealing with subcritical case $p<n$ we have to realize, that the statement cannot be longer that simple. Functions in $W^{s, p}, s p \leq n$, are not necesarily continuous and hence we need to work with the correct representative of our function since the change on a null set may change the dimension of the image. Second and more important obstacle is the fact that it is possible to construct [14] (see also [13]) a continuous mapping even in $W^{1, n}\left((0,1)^{n}, \mathbf{R}^{k}\right)$ such that the image of a zero dimensional set equals $[0,1]^{k}$. This construction is based only on a fact that points have zero capacity in $W^{1, n}$ and hence similar construction can be done also in fractional order Sobolev space $W^{s, p}$, where points have zero capacity. In order to obtain some bound on the dimension of the image we thus need to remove some lower dimensional set where the behaviour can be really "wild".

Theorem 1.2. Let $n, k \in \mathbf{N}, s>0, p>1, s p \leq n$ and $n-s p<\alpha \leq n$. Set

$$
\beta=\frac{p \alpha}{s p-n+\alpha}
$$

Suppose that $f \in W^{s, p}\left(\mathbf{R}^{n}, \mathbf{R}^{k}\right)$ is an $s, p$-quasicontinuous representative. Let us have a set $A$ with $\operatorname{dim}(A) \leq \alpha$. Then there is a $M \subset \mathbf{R}^{n}$ with $\mathcal{H}^{\alpha}(M)=0$ such that $\operatorname{dim}(f(A \backslash M)) \leq \max \{\alpha, \beta\}$. Moreover, if $\beta>n$ then there is a $M \subset \mathbf{R}^{n}$ with $\mathcal{H}^{\alpha}(M)=0$ such that $\operatorname{dim}\left(f\left(\mathbf{R}^{n} \backslash M\right)\right) \leq \beta$.

We also include the following version of the result, which will be proved by the same argument. 
Corollary 1.3. Let $n, k \in \mathbf{N}, s>0, p>1, s p \leq n$ and $n-s p<\alpha \leq n$ and put

$$
\beta=\frac{p \alpha}{s p-n+\alpha} \text {. }
$$

Then there is a set $M \subset \mathbf{R}^{n}$ with $\mathcal{H}^{\alpha}(M)=0$ such that for any set $A \subset \mathbf{R}^{n}$ we have $\operatorname{dim}(f(A \backslash M)) \leq \max \{\operatorname{dim}(A), \beta\}$.

The following example shows that Theorem 1.2 is sharp. In the degenerate case $\alpha \leq n-s p$ the image can have as large dimension as we wish. This degeneracy seems to be connected with the fact that $s, p$-quasicontinuous representatives of the function $f \in W^{s, p}$ are well-defined and have Lebesgue points up to a set of dimension $n-s p$ (see Theorem 2.1 below) and on the set of lower dimension we can have really "bad" behavior even for continuous representative.

Theorem 1.4. Let $n, k \in \mathbf{N}, s>0, p>1, s p \leq n$ and $0<\alpha \leq n$. Set

$$
\beta= \begin{cases}\min \left\{\frac{p \alpha}{s p-n+\alpha}, k\right\} & \text { for } \alpha>n-s p, \\ k & \text { for } \alpha \leq n-s p .\end{cases}
$$

Then there is a continuous mapping $f \in W_{0}^{s, p}\left(\mathbf{R}^{n}, \mathbf{R}^{k}\right)$ and $M_{0} \subset(0,1)^{n}$ with $0<$ $\mathcal{H}^{\alpha}\left(M_{0}\right)<\infty$ such that for every $M \subset(0,1)^{n}$ with $\mathcal{H}^{\alpha}(M)=0$ we have $\operatorname{dim}\left(f\left(M_{0} \backslash\right.\right.$ $M)) \geq \beta$.

\section{Preliminaries}

By $Q(z, r)$ we denote the cube centered at $z \in \mathbf{R}^{d}$ with radius $r>0$.

We use the usual convention that $C$ denotes a generic positive constant whose value may change from line to line.

2.1. Fractional Sobolev spaces. Let us recall the Fourier analytic definition of function spaces. We fix a $C^{\infty}$ function $\phi$ with $\phi(\xi)=1$ for $|\xi| \leq 1$ and $\phi(\xi)=0$ for $\xi \geq 2$. We then find a function $\psi$ such that $\hat{\psi}(\xi)=\phi(\xi)-\phi(2 \xi)$. We define the operator

$$
\Delta_{j} f=\mathcal{F}^{-1}\left(\hat{f}(\xi) \hat{\psi}\left(2^{-j} \xi\right)\right)=f * \psi_{2^{-j}}
$$

where $\psi_{2^{-j}}(x)=2^{j n} \psi\left(2^{j} x\right)$. This scaling is $L^{1}$ homogeneous. Since $\psi$ is a Schwartz function we know that for every $M>0$ there is $C_{M}>0$ such that $|\psi(x)| \leq C_{M}(1+$ $|x|)^{-M}$. Thus for $k>0$

$$
\sup _{x \in B\left(0,2^{-j+k}\right) \backslash B\left(0,2^{-j+k-1}\right)}\left|\psi_{2^{-j}}(x)\right| \leq C_{M} 2^{j n} 2^{-M k} .
$$

Analogous estimate holds for the derivatives and we obtain for example

$$
\sup _{x \in B\left(0,2^{-j+k}\right) \backslash B\left(0,2^{-j+k-1}\right)}\left|\nabla^{2} \psi_{2^{-j}}(x)\right| \leq C_{M} 2^{2 j} 2^{j n} 2^{-M k} .
$$

The function spaces of interest to us are the fractional Sobolev spaces $W^{s, p}, 1<p<$ $\infty, s>0$, where

$$
\|f\|_{W^{s, p}}=\|f\|_{p}+\left\|\left(\sum_{j=-\infty}^{\infty}\left(2^{s j}\left|\Delta_{j} f\right|\right)^{2}\right)^{\frac{1}{2}}\right\|_{p} .
$$


We note that this definition is equivalent to the standard definition found in literature, where the summation starts with $j=0$. This follows by a square function estimate

$$
\left\|\left(\sum_{j=-\infty}^{-1}\left(2^{s j}\left|\Delta_{j} f\right|\right)^{2}\right)^{\frac{1}{2}}\right\|_{p} \leq C\left\|\left(\sum_{j=-\infty}^{-1}\left(\left|\Delta_{j} f\right|\right)^{2}\right)^{\frac{1}{2}}\right\|_{p} \leq C\|f\|_{p} .
$$

We use the modified definition in order to preserve homogeneity in the proof of the positive result. For an introduction to fractional Sobolev spaces, see e.g. [16] and [9, Chapter 6.5].

Let us remind that a function $f$ is $s, p$-quasicontinuous if for every $\varepsilon>0$ we may find an open set $C$ of $(s, p)$-capacity less than $\varepsilon$ such that the function $f$ is continuous outside of $\mathrm{C}$. The folowing is true:

Theorem 2.1. Let $1<p<\infty$ and $0<s p<n$. Let $f \in W^{s, p}\left(\mathbf{R}^{n}\right)$ be an $s, p$-quasicontinuous representative and set

$$
E_{s, p}=\left\{x \in \mathbf{R}^{n}: x \text { is not a Lebesgue point of } f\right\} .
$$

Then $\operatorname{dim}_{\mathcal{H}}\left(E_{s, p}\right) \leq n-s p$.

(See the section 6.1 in [1].)

In the construction of the counterexamples we will need the estimate of the norms of some simple functions (see [11, Lemma 2.3]).

Lemma 2.2. Let $p \in(1, \infty)$ and $s \geq 0$. Let us fix $f_{0} \in C_{c}^{\infty}\left(\mathbf{R}^{n}\right)$ such that

$$
f_{0}=1 \text { on } Q_{0}=(0,1)^{n} \text { and } \operatorname{supp} f_{0} \subset 2 Q_{0} .
$$

A) Let $r \geq 1$. The norm of the function $f_{r}(x)=f_{0}(r x)$ can be estimated by

$$
\left\|f_{r}\right\|_{W^{s, p}} \leq C r^{s-\frac{n}{p}} .
$$

B) Let us fix $N$ disjoint cubes $Q_{i} \subset(0,1)^{n}$ of side length $1 / r$. Let $\tilde{f}_{i}$ denote the translates of $f_{r}$ such that $\tilde{f}_{i} \equiv 1$ on $Q_{i}$ and $\operatorname{supp} \tilde{f}_{i} \subset 2 Q_{i}$ and choose vectors $\xi_{i}$ in the unit ball of $\mathbf{R}^{k}, k \in \mathbf{N}$. Then for the function $f:(0,1)^{n} \rightarrow \mathbf{R}^{k}$ defined as

$$
f(x)=\sum_{i=1}^{N} \tilde{f}_{i}(x) \xi_{i} \text { we have }\|f\|_{W^{s, p}} \leq C N^{\frac{1}{p}}\left\|f_{r}\right\|_{W^{s, p}} \leq C N^{\frac{1}{p}} r^{s-\frac{n}{p}} .
$$

2.2. Probabilistic lemma. In order to prove Theorem 1.4 we will use a probabilistic approach and we will need the following lemma (see [4, Lemma 4.4] for the proof).

Lemma 2.3. Let $\left\{X_{i}\right\}_{i=1}^{\infty}$ be a countable sequence of independent random variables, identically distributed according to the uniform distribution on the unit ball $B$ in $\mathbf{R}^{k}$. Let $c=\left\{c_{i}\right\} \in \ell_{\infty}$ and finally let $0<\beta^{\prime}<k$. Then there is a constant $C$ which depends only on $k$ and $\beta^{\prime}$ so that

$$
\mathbf{E}_{\xi}\left(\left|\sum_{i=1}^{\infty} c_{i} X_{i}\right|^{-\beta^{\prime}}\right) \leq C \rho(c)^{-\beta^{\prime}}
$$

where $\rho(c)$ denotes the second largest value, i.e.

$$
\rho(c)= \begin{cases}\|c\|_{\infty} & \text { if }\|c\|_{\infty}=\sup _{i \in \mathbf{N}}\left|c_{i}\right| \text { is not attained, } \\ \sup _{i \neq i_{0}}\left|c_{i}\right| & \text { if the supremum is attained at } i_{0} .\end{cases}
$$


2.3. Hausdorff and capacitary dimension. Let $\alpha>0$ and $\varepsilon>0$. We use the usual Hausdorff measure of a set $E \subset \mathbf{R}^{d}$, i.e.

$$
\mathcal{H}_{\varepsilon}^{\alpha}(E)=\inf \left\{\sum_{i=1}^{\infty} \operatorname{diam}^{\alpha} A_{i}: E \subset \bigcup_{i=1}^{\infty} A_{i}, \operatorname{diam} A_{i}<\varepsilon\right\} \text { and } \mathcal{H}^{\alpha}(E)=\lim _{\varepsilon \rightarrow 0+} \mathcal{H}_{\varepsilon}^{\alpha}(E) .
$$

The Hausdorff dimension of a set $E$ is

$$
\operatorname{dim}_{\mathcal{H}}(E)=\sup \left\{\alpha>0: \mathcal{H}^{\alpha}(E)=\infty\right\}=\inf \left\{\alpha>0: \mathcal{H}^{\alpha}(E)=0\right\} .
$$

For $\alpha>0$ and $A \subset \mathbf{R}^{k}$, denote by

$$
I_{\alpha}(\mu):=\int_{A} \int_{A}|x-y|^{-\alpha} d \mu(x) d \mu(y)
$$

the $\alpha$-energy of a nonzero finite Radon measure $\mu$ with compact support in $A$. The capacitary dimension of a set $A$ is defined as

$$
\operatorname{dim}_{c}(A)=\sup \left\{\alpha>0: \exists \mu \text { with } I_{\alpha}(\mu)<\infty\right\} .
$$

We will use the well-known fact (see [15, Theorem 8.9]) that the Hausdorff dimension is equal to the capacitary dimension.

2.4. The key lemma. It is well-known the Sobolev space $W^{1, p}, p>n$, is embedded into Hölder continuous functions and moreover, we have the estimate

$$
\int_{B(c, R)}|D f|^{p} \geq C|f(x)-f(y)|^{p} R^{n-p}
$$

for every ball $x, y \in B(c, R)$. In [10, Lemma 3.1] we have shown that similar estimate holds even for $p<n$ on some possibly smaller ball with the additional correction term (see (2.5) below). However for general Sobolev space $W^{k, p}, k>1$ this is not possible if the function $f$ is for example linear on $B(c, R)$. In this case we can have similar estimate on some bigger ball with a different correction term (see (2.6) below). This key estimate was first shown in [11, Lemma 3.1].

Lemma 2.4. Suppose that $a$ and $b$ are Lebesgue points of $f$ in $W^{s, p}\left(\mathbf{R}^{n}\right), 1<$ $p<\infty$ and $s>0$. Let $0<\gamma<p / 2$ and denote $R_{0}=|a-b|$. Then there are $z \in\{a, b\}$ and either $0<R \leq R_{0}$ such that

$$
\int_{B(z, R)}\left(\sum_{j=-\infty}^{\infty}\left(2^{s j}\left|\Delta_{j} f(x)\right|\right)^{2}\right)^{p / 2} d x \geq C_{\gamma}|f(a)-f(b)|^{p} R^{n-s p}\left(R / R_{0}\right)^{\gamma}
$$

or $R_{0} \leq R$ such that

$$
\int_{B(z, R)}\left(\sum_{j=-\infty}^{\infty}\left(2^{s j}\left|\Delta_{j} f(x)\right|\right)^{2}\right)^{p / 2} d x \geq C_{\gamma}|f(a)-f(b)|^{p} R^{n-s p}\left(R / R_{0}\right)^{p-\gamma} .
$$

where the positive constant $C_{\gamma}$ depends only on $\gamma, p, s$ and dimension $n$.

\section{Proof of the supercritical case}

Proof of Theorem 1.1. Suppose $\|f\|_{W^{s, p}} \leq 1$. Let us first discuss the case $\beta>\alpha$. Let us take $\tilde{\alpha}>\alpha$ and

$$
\tilde{\beta}>\frac{p \tilde{\alpha}}{s p-n+\tilde{\alpha}}>\beta
$$


We only need to show that if $\mathcal{H}^{\tilde{\alpha}}(M)=0$ then $\operatorname{dim}(f(M)) \leq \tilde{\beta}$. We choose a $0<\varepsilon<1$ and take a covering of $M$ by balls $B\left(z_{j}, t_{j}\right)$ such that $\sum t_{j}^{\tilde{\alpha}}<\varepsilon$. We refine the covering using a stopping time argument. We consider the dyadic cubes $Q$ such that if $l=\operatorname{diam}(Q)$ and $y$ is the center of $Q$ then

$$
\sum_{\left\{j: z_{j} \in Q \text { and } B\left(t_{j}, z_{j}\right) \subset B(y, 10 l)\right\}} t_{j}^{\tilde{\alpha}} \geq l^{\tilde{\alpha}} .
$$

We assume that $z_{j}$ never falls to the boundary of $Q$, obviously we may always modify the covering to satisfy this. We select all the maximal dyadic cubes $Q_{i}$ with this property and then we replace the original covering by a new covering

$$
\left\{B\left(x_{i}, r_{i}\right) ; r_{i}=10 \operatorname{diam}\left(Q_{i}\right), x_{i} \text { is the center of } Q_{i}\right\} .
$$

It is obvious that

$$
\bigcup_{j} B\left(t_{j}, z_{j}\right) \subset \bigcup_{i} B\left(x_{i}, r_{i}\right)
$$

and

$$
\sum_{i} r_{i}^{\tilde{\alpha}} \leq C \varepsilon
$$

Moreover, the new covering also has the property that whenever we choose $x$ and $R>0$ we get

$$
\sum_{B\left(x_{i}, r_{i}\right) \subset B(x, R)} r_{i}^{\tilde{\alpha}} \leq C R^{\tilde{\alpha}}
$$

this follows from the choice of maximal dyadic cubes in the construction. Denote $\rho_{i}=\operatorname{diam}\left(f\left(B_{i}\left(x_{i}, r_{i}\right)\right)\right)$. As $f$ is continuous (since $f \in W^{s, p}$ for $s p>n$ ) we may assume that $r_{i} \leq 1$ and $\rho_{i} \leq 1$. Without loss of generality we may assume that $\sum_{i} \rho_{i}^{\tilde{\beta}} \geq 1$, otherwise $\operatorname{dim}(f(M)) \leq \tilde{\beta}$ and the proof is over. We claim that for every $0<\gamma<1$ there are some $0<\delta, \sigma<1$ such that

$$
\sum_{\delta<r_{i} \leq 2 \delta, \sigma<\rho_{i} \leq 2 \sigma} \rho_{i}^{\tilde{\beta}}>C_{\gamma} \delta^{\gamma} \sigma^{\gamma} .
$$

Otherwise we easily get a contradiction from

$$
1 \leq \sum_{i} \rho_{i}^{\tilde{\beta}} \leq \sum_{\left\{a \in \mathbf{N}: 2^{-a}<r_{i} \leq 2^{-a+1}\right\}} \sum_{\left\{b \in \mathbf{N}: 2^{-b}<\rho_{i} \leq 2^{-b+1}\right\}} \rho_{i}^{\beta} \leq C_{\gamma} \sum_{a=1}^{\infty} \sum_{b=1}^{\infty} 2^{-a \gamma} 2^{-b \gamma} .
$$

We choose the precise value of $\gamma$ later. We fix the $\sigma$ and $\delta$ with property (3.4) and reduce the family of the points $x_{i}$ accordingly. The family now has $K$ elements and

$$
K \geq C_{\gamma} \delta^{\gamma} \sigma^{\gamma-\tilde{\beta}}
$$

while also $K \delta^{\tilde{\alpha}} \leq C \varepsilon$. Therefore, we get the relation (as $\left.\varepsilon<1\right)$

$$
\delta^{-\tilde{\alpha}-\gamma} \geq C \sigma^{\gamma-\tilde{\beta}}
$$

We now invoke the Lemma 2.4. We take two Lebesgue points of $f$ denoted as $x, x^{\prime} \in B_{i}\left(x_{i}, r_{i}\right)$ such that $\left|f(x)-f\left(x^{\prime}\right)\right| \geq \rho_{i} / 4$ and $\left|x-x^{\prime}\right| \geq r_{i}$ and we put $R_{0}=\left|x-x^{\prime}\right|$. For small enough $\gamma$ we have $n-s p+\gamma<0$ and hence (2.5) remains 
valid also for $R=R_{0}$. This shows that we can always assume that $R \geq R_{0}$ and use (2.6). We get that there is a ball centered at $z=x$ or $z=x^{\prime}$ and $R \geq 3 R_{0}$ such that

$$
\left|f(x)-f\left(x^{\prime}\right)\right|^{p} \leq C R^{s p-n}\left(R_{0} / R\right)^{p-\gamma} \int_{B(z, R)}\left(\sum_{j=-\infty}^{\infty}\left(2^{s j}\left|\Delta_{j} f(x)\right|\right)^{2}\right)^{p / 2} d x .
$$

Moreover, we see that if $R \leq \delta$ and $\gamma<p$ the estimate remains valid for $R=\delta$. We therefore assume $R \geq \delta$. We denote these balls $B\left(z_{j}, R_{j}\right)$ and using Vitali covering we pass to a disjoint subcollection indexed by $k$ such that

$$
\bigcup_{j} B\left(z_{j}, R_{j}\right) \subset \bigcup_{k} B\left(z_{k}, 5 R_{k}\right) .
$$

By $r_{i} \geq \delta$ (see (3.4)) and (3.3) we know that each of the balls

$$
B\left(z_{k}, 5 R_{k}\right) \text { contains at most } C\left(R_{k} / \delta\right)^{\tilde{\alpha}} \text { of the points } x_{i} \text {. }
$$

For $j \in \mathbf{N}$ (recall $R_{k} \geq \delta$ ) we consider

$$
S_{j}:=\left\{x_{i}: x_{i} \in B\left(z_{k}, 5 R_{k}\right) \text { and } 2^{j}<R_{k} / \delta \leq 2^{j+1}\right\} .
$$

Using Hölder inequality $(\# S=$ number of elements of a set $S$ )

$$
\left(\sup _{j \in \mathbf{N}} 2^{j \gamma} \# S_{j}\right) \sum_{j=1}^{\infty} 2^{-j \gamma} \geq \sum_{j=1}^{\infty} \# S_{j} \geq \#\left\{x_{i}\right\}=K
$$

we get that there is a $j$ such that $\# S_{j}>C 2^{-j \gamma} K$. This and (3.8) give that

$$
\#\left\{z_{k}: 2^{j}<R_{k} / \delta \leq 2^{j+1}\right\} \geq C \frac{2^{-j \gamma} K}{\left(R_{k} / \delta\right)^{\tilde{\alpha}}} \geq C 2^{-j(\tilde{\alpha}+\gamma)} K .
$$

Finally by $\|f\|_{W^{s, p}} \leq 1,(3.7),\left|f(x)-f\left(x^{\prime}\right)\right| \sim \rho_{i} \sim \sigma_{i}, R_{0}=\left|x-x^{\prime}\right| \sim r_{i} \sim \delta$ (where we used (3.4)) and (3.5) we have

$$
\begin{aligned}
1 & \geq \int_{\bigcup_{\left\{k ; 2^{j}<R_{k} / \delta \leq 2^{j+1}\right\}} B\left(z_{k}, R_{k}\right)}\left(\sum_{l=-\infty}^{\infty}\left(2^{s l}\left|\Delta_{l} f(x)\right|\right)^{2}\right)^{p / 2} d x \\
& \geq C \sigma^{p}\left(2^{j} \delta\right)^{n-s p} 2^{j(\gamma-p)} 2^{-j(\tilde{\alpha}+\gamma)} K \\
& \geq C \sigma^{p-\tilde{\beta}+\gamma}\left(2^{j} \delta\right)^{n-s p} 2^{j(-\gamma+p)} 2^{-j(\tilde{\alpha}+\gamma)} \delta^{\gamma} \\
& \geq C 2^{j(n-s p-\tilde{\alpha}+p-2 \gamma)} \sigma^{p-\tilde{\beta}+\gamma} \delta^{n-s p+\gamma} .
\end{aligned}
$$

We first observe that $\beta=\frac{p \alpha}{s p-n+\alpha}>\alpha$ implies that $n-s p-\tilde{\alpha}+p-2 \gamma>0$ for small $\gamma$ and suitable $\tilde{\alpha}$ close to $\alpha$ and together with $s p>n$ also $p-\tilde{\beta}+\gamma>0$ for suitable $\tilde{\beta}$ close to $\beta$ and any $\gamma>0$. Therefore, by (3.6) we may use $\sigma \geq C \delta^{\frac{\tilde{\alpha}+\gamma}{-\gamma+\beta}}$ to obtain

$$
1 \geq C \delta^{(p-\tilde{\beta}+\gamma) \frac{\tilde{\alpha}+\gamma}{-\gamma+\tilde{\beta}}} \delta^{n-s p+\gamma} .
$$

The last exponent is negative if we take $\gamma=0$ by (3.1) and therefore it is also negative for a small $\gamma$. We have $\delta \rightarrow 0$ as $\varepsilon \rightarrow 0$, which is a contradiction. This finishes the case $\beta>\alpha$.

In case $\beta \leq \alpha$ we make the following preliminary observation: if $(s-1) p-n>0$, then $W^{s, p}$ is imbedded into Lipschitz functions and the statement is trivial. Therefore, we assume the opposite. For the most part, we repeat the previous argument. The value of $\beta$ is not relevant to us and is effectively replaced by $\alpha$. Therefore, we take 
$\alpha<\tilde{\alpha}<\tilde{\beta}$ and proceed to obtain the same coverings as before. Set $R_{\max }=\varepsilon^{1 / \tilde{\alpha}}$. We obtain (3.9) for some $j$ and this inequality is usefull only if $2^{j} \leq R_{\max } / \delta$ and in this case we obtain the inequality (3.10) as before, or there is at least one $R_{k} \geq R_{\max }$. In that case, we get use $(s-1) p-n+\gamma<0$ for small $\gamma$ and (2.6) to obtain

$$
\begin{aligned}
1 & \geq \int_{B\left(z_{k}, R_{k}\right)}\left(\sum_{l=-\infty}^{\infty}\left(2^{s l}\left|\Delta_{l} f(x)\right|\right)^{2}\right)^{p / 2} d x \\
& \geq C \sigma^{p} R_{k}^{n-s p}\left(R_{k} / R_{0}\right)^{p-\gamma} \geq \sigma^{p} R_{\max }^{n-s p}\left(R_{\max } / R_{0}\right)^{p-\gamma} .
\end{aligned}
$$

Therefore, we take $\tilde{j}=j$ if for all $k$ we have $R_{k} \leq 2 R_{\max }$ and $\tilde{j}=\log _{2}\left(R_{\max } / \delta\right)$ otherwise. As $2^{j} \leq R_{\max } / \delta$ in the first case we always have

$$
2^{\tilde{j}} \leq \frac{R_{\max }}{\delta}
$$

In the second case $\left(R_{k} \geq R_{0}\right.$ for some $\left.k\right),(3.2), r_{i} \sim \delta, \tilde{j}=\log _{2}\left(R_{\max } / \delta\right)$ and $R_{\max }=\varepsilon^{1 / \tilde{\alpha}}$ imply that $K<C 2^{\tilde{j} \tilde{\alpha}}$. Now $\tilde{j}=\log _{2}\left(R_{\max } / \delta\right)$ and $\delta \geq R_{0}=\left|x-x^{\prime}\right|$ give us

$$
R_{\max }^{n-s p}\left(R_{\max } / R_{0}\right)^{p-\gamma} \geq K 2^{-\tilde{j} \tilde{\alpha}} 2^{\tilde{j}(n-s p+p-\gamma)} \delta^{n-s p} .
$$

Therefore with the help of (3.5) we have in both cases the inequality (3.10) as

$$
\begin{aligned}
1 & \geq \int_{\bigcup_{\left\{k ; 2^{j}<R_{k} / \delta \leq 2^{j+1}\right\}} B\left(z_{k}, R_{k}\right)}\left(\sum_{l=-\infty}^{\infty}\left(2^{s l}\left|\Delta_{l} f(x)\right|\right)^{2}\right)^{p / 2} d x \\
& \geq C 2^{\tilde{j}(n-s p-\tilde{\alpha}+p-2 \gamma)} \sigma^{p-\tilde{\beta}+\gamma} \delta^{n-s p+\gamma} .
\end{aligned}
$$

Now we split cases. If $p>\alpha$, we use (3.6) and we obtain

$$
1 \geq C 2^{\tilde{j}(n-s p-\tilde{\alpha}+p-2 \gamma)} \delta^{(p-\tilde{\beta}+\gamma) \frac{\tilde{\alpha}+\gamma}{-\gamma+\tilde{\beta}}} \delta^{n-s p+\gamma} .
$$

For $\gamma=0$ and $\tilde{\beta}=\alpha$ the power of $\delta$ and $2^{\tilde{j}}$ is the same and negative (recall that $\beta=\frac{p \alpha}{s p-n+\alpha}<\alpha$ ). For very small $\gamma$ with $\gamma<<\tilde{\beta}-\tilde{\alpha}<<1$ this gives with the help of (3.12) that

$$
1 \geq C R_{\max }^{(n-s p-\tilde{\alpha}+p-2 \gamma)} \delta^{-\bar{\gamma}}
$$

where the constant $\bar{\gamma}$ is positive if $\gamma$ is chosen small enough. Now we see that for $\varepsilon \rightarrow 0$ the right hand side of the inequality goes to infinity as $R_{\max }=\varepsilon^{1 / \tilde{\alpha}}$, a contradiction.

If we assume $p \leq \alpha$, the estimate (2.6) gives (here we may select any $R_{k}$ from the covering and we use the fact that the integral is at most 1)

$$
\sigma^{p} \leq C R_{k}^{s p-n}\left(\delta / R_{k}\right)^{p-\gamma}
$$

and therefore by (3.12) and $s p-n-p \geq s p-n-\alpha \geq 0$

$$
\left(2^{\tilde{j}} \sigma\right)^{p} \leq C R_{\max }^{s p-n} 2^{p(\tilde{j}-j)-\gamma \tilde{j}} \leq C R_{\max }^{s p-n}
$$

as $\tilde{j} \leq j$. Since for small $\gamma$ we have $p-\tilde{\beta}+\gamma<0$ we can use (3.13), (3.14) and (3.12) to obtain

$$
\begin{aligned}
1 & \geq C 2^{\tilde{j}(n-s p-\tilde{\alpha}+p-2 \gamma)} \sigma^{(p-\tilde{\beta}+\gamma)} \delta^{n-s p+\gamma} \\
& \geq C 2^{\tilde{j}(-\tilde{\alpha}+\tilde{\beta}+4 \gamma)} R_{\max }^{(p-\tilde{\beta}+\gamma)(s p-n) / p} R_{\max }^{n-s p+\gamma} \geq C R_{\max }^{(p-\tilde{\beta}+\gamma)(s p-n) / p} R_{\max }^{n-s p+\gamma}
\end{aligned}
$$

and the result now follows, as the exponent is negative $(s p<n$ and $p-\tilde{\beta}+\gamma<0)$ and $R_{\max } \rightarrow 0$ with $\varepsilon \rightarrow 0$. 


\section{Proof of the subcritical case}

Let us introduce dyadic cubes on $\mathbf{R}^{n}$. For $w \in \mathbf{Z}$ we denote $\mathcal{D}_{w}=\left\{Q_{i}\right\}$ where $Q_{i}$ are closed cubes with vertices in the points $2^{-w} \mathbf{Z}^{n} \cap[0,1]^{n}$ and with volume $2^{-w n}$.

Proof of Theorem 1.2. Let us first observe that the value of the fraction defining $\beta$ is decreasing in $s$ and approaches $\infty$ as $s p \rightarrow(n-\alpha)+$. Therefore the case $\beta<\alpha$ may be treated by the trivial imbedding of $W^{s, p}$ into $W^{s^{\prime}, p}$ for $s^{\prime} \leq s$, we just replace $s$ by some smaller $s^{\prime}$ for which $\frac{p \alpha}{s^{\prime} p-n+\alpha}=\alpha$.

Let us suppose that $\alpha \leq \beta,\|f\|_{s, p} \leq 1$ and take $\tilde{\beta}>\beta$. Let us first treat the case $\beta \geq n$. As $\beta=\frac{p \alpha}{s p-n+\alpha}$ and $\tilde{\beta}>\beta$ we can fix some small $\varepsilon>0$ such that

$$
\begin{aligned}
& \frac{\tilde{\beta}}{p} \varepsilon+\alpha-\frac{\tilde{\beta}}{p}(\alpha+s p-n)<0 \\
& \text { and } \quad \beta-\tilde{\beta}+2 \varepsilon \frac{2 \tilde{\beta}}{p}<0 .
\end{aligned}
$$

For $k \in \mathbf{N} \cup\{0\}$ and $w \in \mathbf{Z}$ we denote the system of cubes where the norm is big as

$$
\begin{aligned}
& D_{w}^{\varepsilon, k}=\left\{Q \in D_{w}: \int_{Q}\left(\sum_{j=-\infty}^{\infty}\left(2^{s j}\left|\Delta_{j} f(x)\right|\right)^{2}\right)^{p / 2} d x \geq 2^{-w(\alpha+(k-1) \varepsilon)}\right\}, \\
& M_{w}^{\varepsilon, 0}=\bigcup_{Q \in D_{w}^{\varepsilon, 0}} 5 Q \text { and } M_{w}^{\varepsilon, k}=\bigcup_{Q \in D_{w}^{\varepsilon, k} \backslash D_{w}^{\varepsilon, k-1}} 5 Q \text { for } k \geq 1,
\end{aligned}
$$

where $5 Q$ is the cube with the same center and orientation inflated 5 times. We work with the Cantor type set

$$
\tilde{M}_{j}^{\varepsilon, k}=\bigcup_{j \leq w} M_{w}^{\varepsilon, k}
$$

and

$$
M^{\varepsilon, k}=\bigcap_{j \in \mathbf{Z}} \tilde{M}_{j}^{\varepsilon, k}
$$

From $\|f\|_{s, p} \leq 1,(2.3)$ and (4.3) we obtain that the set $M_{w}^{\varepsilon, 0}$ may be covered by $2^{w(\alpha-\varepsilon)}$ balls of the diameter $5 \sqrt{n} 2^{-w}$ and therefore $\mathcal{H}^{\alpha}\left(M^{\varepsilon, 0}\right)=0$.

Let us first handle the case $\beta=\frac{p \alpha}{s p-n+\alpha}>n$. Since $n-s p \leq \alpha<n$ we can fix $K$ such that

$$
\frac{p}{\alpha+(K-1) \varepsilon+s p-n}>1 \text { but } \frac{p}{\alpha+K \varepsilon+s p-n} \leq 1
$$

Our exceptional set $M$ is the union of $M^{\varepsilon, 0}$ with non-Lebesgue points of $f$. We want to estimate the dimension of the Lebesgue points of $f$ in the set $f\left(\mathbf{R}^{n} \backslash M^{\varepsilon, 0}\right)$. The set $f\left(\mathbf{R}^{n} \backslash M\right)$ is covered by sets

$$
f\left(M^{\varepsilon, 1} \backslash M^{\varepsilon, 0}\right), f\left(M^{\varepsilon, 2} \backslash\left(M^{\varepsilon, 1} \cup M^{\varepsilon, 0}\right)\right), \ldots, f\left(\mathbf{R}^{n} \backslash \cup_{0 \leq k<K} M^{\varepsilon, k}\right)
$$

therefore it is enough to estimate the dimension of each of these sets individually. Let us recall that the set of non-Lebesque points of our $s, p$-quasicontinuous representative has dimension smaller or equal to $n-s p$ (see Theorem 2.1) and as $n-s p<\alpha$ we can add these points to the set $M$ without increasing its dimension bound. 
First we choose $0 \leq k<K, j \geq 1$ and then we choose an integer $l \sim \log j$. We have

$$
M^{\varepsilon, k} \subset \tilde{M}_{j}^{\varepsilon, k}=\bigcup_{j \leq w} \bigcup_{Q \in D_{w}^{\varepsilon, k} \backslash D_{w}^{\varepsilon, k-1}} 5 Q .
$$

For a fixed $w \geq j$ we pick one of the cubes $5 Q$ in the above union and estimate the diameter of $f\left(5 Q \backslash \cup_{u \leq k-1} \tilde{M}_{l}^{\varepsilon, u}\right)$ using Lemma 2.4. Let us take two Lebesgue points of $f$ denoted as $x, x^{\prime} \in 5 Q \backslash \cup_{u \leq k-1} \tilde{M}_{l}^{\varepsilon, u}$ and consider a ball $B(x, r)$. In case $r<2^{-l} n^{-1 / 2}$ we choose integer $v \approx \log _{2} r$ such that $-v \geq l$ and since

$$
x \notin \bigcup_{u \leq k-1} \tilde{M}_{l}^{\varepsilon, u}=\bigcup_{u \leq k-1} \bigcup_{l \leq w} \bigcup_{Q \in D_{w}^{\varepsilon, u} \backslash D_{w}^{\varepsilon, u-1}} 5 Q=\bigcup \bigcup_{l \leq w} 5 Q
$$

we can find a cube $Q \in D_{-v}$ (of side length $2^{v}$ ) such that $B(x, r) \subset Q$ and $Q \notin D_{-v}^{\varepsilon, k-1}$. It follows that

$$
\int_{B(x, r)}\left(\sum_{j=-\infty}^{\infty}\left(2^{s j}\left|\Delta_{j} f(x)\right|\right)^{2}\right)^{p / 2} d x \leq 2^{v(\alpha+(k-2) \varepsilon)}
$$

while for the larger $r\left(r \geq 2^{-l} n^{-1 / 2}\right)$ we get simply

$$
\int_{B(x, r)}\left(\sum_{j=-\infty}^{\infty}\left(2^{s j}\left|\Delta_{j} f(x)\right|\right)^{2}\right)^{p / 2} d x \leq 1 .
$$

Similar estimates hold for $x^{\prime}$. We denote $\left|x-x^{\prime}\right|=R_{0}$. Combining the estimates (2.5), (2.6), (4.7), (4.8) we get

$$
\left|f(x)-f\left(x^{\prime}\right)\right| \leq \max \left\{A_{1}, A_{2}, A_{3}\right\}
$$

where $A_{1}$ corresponds to the case $r<R_{0}, A_{2}$ to $R_{0}<r<2^{-l} n^{-1 / 2}$ and $A_{3}$ to $2^{-l} n^{-1 / 2} \leq r$.

The quantity $A_{1}$ represents the situation when $r<R_{0}$, therefore we also have $-v>C w$ (recall that $x, x^{\prime} \in 5 Q$ for some $Q \in D_{w}$ ). We use (2.5), (4.7) and $r \sim 2^{v}$ to obtain

$$
A_{1}^{p} \leq \sup _{r<R_{0}} C 2^{v(\alpha+(k-2) \varepsilon)} r^{s p-n}\left(R_{0} / r\right)^{\gamma} \leq \sup _{r<R_{0}} C r^{\alpha+(k-2) \varepsilon} r^{s p-n}\left(R_{0} / r\right)^{\gamma} .
$$

As $n-s p<\alpha$ we can assume that $0<\gamma<\alpha+s p-n$ and the supremum is attained for $r=R_{0}$ and with the help of $R_{0} \sim 2^{-w}$ we get

$$
A_{1}^{p} \leq C 2^{-w(\alpha+(k-2) \varepsilon+s p-n)} .
$$

The quantity $A_{2}$ is reserved for the case $R_{0} \leq r<2^{-l} n^{-1 / 2}$, where we may still use (4.7), but we need to use (2.6) and we obtain

$$
\begin{aligned}
A_{2}^{p} & \leq \sup _{R_{0} \leq r<2^{-l} n^{-1 / 2}} C 2^{v(\alpha+(k-2) \varepsilon)} r^{s p-n}\left(R_{0} / r\right)^{p-\gamma} \\
& \leq \sup _{R_{0} \leq r<2^{-l} n^{-1 / 2}} C r^{\alpha+(k-2) \varepsilon} r^{s p-n}\left(R_{0} / r\right)^{p-\gamma} .
\end{aligned}
$$

By (4.4) and $0 \leq k<K$ we have

$$
\frac{p}{\alpha+(k-2) \varepsilon+s p-n} \geq \frac{p}{\alpha+(K-1) \varepsilon+s p-n}>1
$$


and we obtain that the power of $r$ is negative (for fixed but small enough $\gamma$ ) and hence the supremum is attained for $r=R_{0}$ and with the help of $R_{0} \sim 2^{-w}$ we get

$$
A_{2}^{p} \leq C 2^{-w(\alpha+(k-2) \varepsilon+s p-n)} .
$$

In the case $r \geq 2^{-l} n^{-1 / 2}$ we estimate $A_{3}$ using (4.8) and (2.6) as

$$
A_{3}^{p} \leq \sup _{r \geq 2^{-l} n^{-1 / 2}} C r^{s p-n}\left(R_{0} / r\right)^{p-\gamma} \leq C R_{0}^{p-\gamma} 2^{-l(s p-n-p+\gamma)},
$$

where in the last inequality we used that $s p-n-p<0$ by (4.10). We have chosen $l \approx \log j$ (recall that $j \leq w$ by (4.6)) and it follows that the terms with $2^{-l}$ are very small and thus we get

$$
A_{3}^{p} \leq C 2^{-w(p-2 \gamma)} .
$$

From $\|f\|_{s, p} \leq 1$ we can see that for a fixed $w$ there are at most $2^{w(\alpha+(k-1) \varepsilon)}$ cubes $Q_{i}$ in $D_{w}^{\varepsilon, k}$. We denote

$$
u_{i}=\operatorname{diam} f\left(5 Q_{i} \backslash \bigcup_{u \leq k-1} \tilde{M}_{l}^{\varepsilon, u}\right)
$$

and proceed to estimate

$$
\sum_{Q_{i} \in D_{w}^{\varepsilon, k}} u_{i}^{\tilde{\beta}} \leq 2^{w(\alpha+(k-1) \varepsilon)} \max \left\{A_{1}, A_{2}, A_{3}\right\}^{\tilde{\beta}} .
$$

By (4.10) we can see that the estimate of $A_{3}$ is better than the estimate of $A_{1}$ and $A_{2}$. This gives

$$
\sum_{Q_{i} \in D_{w}^{\varepsilon, k}} u_{i}^{\tilde{\beta}} \leq C 2^{w(\alpha+(k-1) \varepsilon)-w \frac{\tilde{\beta}}{p}(\alpha+(k-2) \varepsilon+s p-n)} .
$$

As $s p<n$ it is easy to check that $p<\beta=\frac{p \alpha}{s p-n+\alpha}<\tilde{\beta}$ and with (4.1) this implies that the power of $2^{w}$ in the previous expression is negative as it equals to

$$
(k-1) \varepsilon-\frac{\tilde{\beta}}{p}(k-1) \varepsilon+\frac{\tilde{\beta}}{p} \varepsilon+\alpha-\frac{\tilde{\beta}}{p}(\alpha+s p-n) .
$$

By (4.6) we may sum over $w \geq j$ to get

$$
\mathcal{H}^{\tilde{\beta}}\left(f\left(M_{j}^{\varepsilon, k} \backslash \bigcup_{0 \leq u<k-1} M_{l}^{\varepsilon, u}\right)\right) \leq C(j),
$$

where $C(j) \rightarrow 0$ as $j \rightarrow \infty$. Let us fix $l_{0} \in \mathbf{N}$ and choose $j_{0}$ such that $\log j_{0}>10 l_{0}$ (recall $l \sim \log j$ ). Now

$$
M^{\varepsilon, k} \backslash \bigcup_{0 \leq u<k-1} M_{l_{0}}^{\varepsilon, u}=\bigcap_{j=j_{0}}^{\infty}\left(M_{j}^{\varepsilon, k} \backslash \bigcup_{0 \leq u<k-1} M_{l_{0}}^{\varepsilon, u}\right) \subset \bigcap_{j=j_{0}}^{\infty}\left(M_{j}^{\varepsilon, k} \backslash \bigcup_{0 \leq u<k-1} M_{l}^{\varepsilon, u}\right) .
$$

By (4.13) we get that $\mathcal{H}^{\beta}$ of the image of this set is 0 and in the limit $l_{0} \rightarrow \infty$ we get

$$
\mathcal{H}^{\tilde{\beta}}\left(f\left(M^{\varepsilon, k} \backslash \bigcup_{0 \leq u<k-1} M^{\varepsilon, u}\right)\right)=0 .
$$

In view of (4.5) it remains to consider the set $\mathbf{R}^{n} \backslash \bigcup_{0 \leq k<K} M^{\varepsilon, k}$. We consider only the part of this set which is containded in the unit cube, the other parts are handled 
the same way. We cover this set with all $2^{w n}$ cubes from $D_{w}$. Similarly as in (4.12) we obtain the estimate

$$
\sum_{Q_{i} \in D_{w}^{\varepsilon, k}} u_{i}^{\tilde{\beta}} \leq C 2^{w n} 2^{-w \frac{\tilde{\beta}}{p}(\alpha+(K-2) \varepsilon+s p-n)} .
$$

As $\tilde{\beta}>\beta \geq n$ we obtain from the second inequality in (4.4) and (4.2) that the power of $2^{w}$ is again negative as

$n-\tilde{\beta}+\tilde{\beta}-\frac{\tilde{\beta}}{p}(\alpha+(K-2) \varepsilon+s p-n) \leq\left[\beta-\tilde{\beta}+\frac{2 \varepsilon \tilde{\beta}}{p}\right]+\frac{\tilde{\beta}}{p}[p-(\alpha+K \varepsilon+s p-n)]$

and we may proceed similarly as above to get our conclusion.

Let $\beta=\frac{\alpha p}{\alpha+s p-n}<n$ and choose $\tilde{\alpha}>\alpha$ close enough so that

$$
\eta:=\tilde{\alpha}-\frac{\tilde{\beta}}{p}(\alpha-2 \varepsilon+s p-n)<0 .
$$

We proceed as above, but we only consider the sets $A$ and $M^{\varepsilon, 0}$. We may assume that $A$ is a subset of unit cube. We estimate only the dimension of the set $f\left(A \backslash M^{\varepsilon, 0}\right)$ and this is similar to the previous estimate of $f\left(M^{\varepsilon, 1} \backslash M^{\varepsilon, 0}\right)$. Let us fix $j \in \mathbf{N}$ and $l \sim \log j$. Instead of the covering (4.6) we cover $A$ by dyadic cubes $\left\{Q_{i}\right\}_{i=1}^{a}$ such that $Q_{i}$ has side length $2^{-w_{i}} \leq 2^{-j}$ and

$$
\sum_{i} \operatorname{diam}^{\tilde{\alpha}}\left(Q_{i}\right)<1 \text { and hence } \#\left\{i: w_{i}=w\right\} \leq 2^{\tilde{\alpha} w} .
$$

We consider two Lebesgue points $x, x^{\prime} \in Q_{i} \backslash \tilde{M}_{l}^{\varepsilon, 0}$ and consider a ball $B(x, r)$. As before we have (4.9) and analogously to the proof of (4.11) we obtain for every $w \geq j$

$\sum_{\left\{i: w_{i}=w\right\}} \operatorname{diam}^{\tilde{\beta}} f\left(Q_{i} \backslash \tilde{M}_{l}^{\varepsilon, 0}\right) \leq 2^{w \tilde{\alpha}} \max \left\{A_{1}, A_{2}, A_{3}\right\}^{\tilde{\beta}} \leq C 2^{w \tilde{\alpha}-w \frac{\tilde{\beta}}{p}(\alpha-2 \varepsilon+s p-n)}=C 2^{w \eta}$.

By (4.14) we may sum this over all $w \geq j$ and finish the proof as before.

To prove the corollary, we define the set $M$ as before. We choose $K$ so that (4.4) holds, but also choose $K^{\prime}$ so that for $k<K^{\prime}$ we have $\alpha+(k-1) \varepsilon<\operatorname{dim}(A)$ but $\alpha+\left(K^{\prime}-1\right) \varepsilon \geq \operatorname{dim}(A)$. We then put $\tilde{K}=\min \left(K, K^{\prime}\right)$. The set $f(A \backslash M)$ is covered by

$$
f\left(M^{\varepsilon, 1} \backslash M^{\varepsilon, 0}\right), f\left(M^{\varepsilon, 2} \backslash\left(M^{\varepsilon, 1} \cup M^{\varepsilon, 0}\right)\right), \ldots, f\left(A \backslash \bigcup_{0 \leq k<\tilde{K}^{2}} M^{\varepsilon, k}\right) .
$$

We estimated the dimension of all these terms except the last one. However, it is obvious that the dimension of $f\left(A \backslash \bigcup_{0 \leq k<\tilde{K}} M^{\varepsilon, k}\right)$ may be estimated using the same method as in the previous paragraph

$$
\sum_{\left\{i: w_{i}=w\right\}} \operatorname{diam}^{\tilde{\beta}} f\left(Q_{i} \backslash \tilde{M}_{l}^{\varepsilon, 0}\right) \leq C 2^{w(\operatorname{dim} A+\varepsilon)} 2^{-w \frac{\tilde{\beta}}{p}(\alpha+(\tilde{K}-2) \varepsilon+s p-n)}
$$

and with the help of $\alpha+\left(K^{\prime}-1\right) \varepsilon \geq \operatorname{dim}(A)$ we easily show that the exponent is negative for $K^{\prime} \leq K$. For $K<K^{\prime}$ and $\operatorname{dim} A \leq \beta$ we use (4.4) to show that the exponent in (4.16) is negative. For $\beta<\operatorname{dim} A$ we may argue as at the very beginning of the proof and by choosing $s^{\prime} \leq s$ we can assume that this cannot happen. This proves the corollary. 


\section{Counterexample}

For the construction of the counterexample in Theorem 1.4 we use the approach that was developed in [4, Theorem 1.4], [12, Theorem 3] and [11, Theorem 1.2]. The function $f$ has wild behavior on a regular $\alpha$-dimensional set and in the proof we need a measure on the image of this set (pushforward of the $\alpha$-dimensional Hausdorff measure) and then we use the definition of capacitary dimension which equals the Hausdorff dimension. For the convenience of the reader we include all details.

Proof of Theorem 1.4. First we will treat the nondegenerate case $\alpha>n-s p$ and $\beta \leq k$ and at the end we briefly mention how to adjust the construction for other cases.

Let us denote $Q_{0}=(0,1)^{n}$ and by $\mathcal{Q}_{j}, j \in \mathbf{N}$ we denote the system of $2^{j n}$ dyadic cubes of side length $2^{-j}$ in $Q_{0}$. We fix a system of cubes $\mathcal{A}_{j} \subset \mathcal{Q}_{j}$ such that its cardinality satisfies $([a]$ denotes integer part)

$$
\# \mathcal{A}_{j}=\left[2^{j \alpha}\right]
$$

and

$$
\text { for every } Q \in \mathcal{A}_{j+1} \text { there is } \tilde{Q} \in \mathcal{A}_{j} \text { such that } Q \subset \tilde{Q} \text {. }
$$

We denote $\mathcal{A}=\bigcup_{j \in \mathbf{N}} \mathcal{A}_{j}$. It is easy to check that the Hausdorff dimension of the set

$$
M_{0}:=\bigcap_{j=1}^{\infty} \bigcup_{Q \in \mathcal{A}_{j}} Q .
$$

equals $\alpha$ and $0<\mathcal{H}^{\alpha}\left(M_{0}\right)<\infty$. Moreover, we can and will require that the cubes are evenly distributed in the next steps so that there is $0<C_{0}$ such that

$$
\text { for each } j \in \mathbf{N} \text { and } Q \in \mathcal{A}_{j} \text { we have } \mathcal{H}^{\alpha}\left(Q \cap M_{0}\right) \leq C_{0} 2^{-j \alpha} \text {. }
$$

Let $M \subset(0,1)^{n}$ satisfy $\mathcal{H}^{\alpha}(M)=0$ as in the assumption of the theorem.

For each $\xi$ we describe later, we will construct a map $f_{\xi} \in W^{s, p}\left(\mathbf{R}^{n}, \mathbf{R}^{k}\right)$ that satisfies

$$
\mathcal{H}^{\beta^{\prime}}\left(f_{\xi}\left(M_{0} \backslash M\right)\right)=\infty
$$

almost surely in $\xi$, for each $\beta^{\prime}<\beta$, and thus we will get our conclusion.

Let us fix $f_{0} \in C_{c}^{\infty}\left(\mathbf{R}^{n}\right)$ such that $f_{0}=1$ on $Q_{0}, 0 \leq f_{0} \leq 1$ and $\operatorname{supp} f_{0} \subset 2 Q_{0}$. For each $Q \in \mathcal{A}_{j}, j \in \mathbf{N}$, let $\psi_{Q} \in C_{0}^{\infty}\left(\mathbf{R}^{n}\right)$ be a function defined as an appropriate translate of $f_{2^{j}}$ from Lemma 2.2. That is $\psi_{Q}$ is supported in the cube $2 Q$ of side length $2^{-j+1}$ and

(i) $0 \leq \psi_{Q} \leq 1$

(ii) $\psi_{Q} \equiv 1$ on $Q$,

(iii) $\psi_{Q} \equiv 0$ on the complement of $2 Q$,

(iv) $\left\|\psi_{Q}\right\|_{W^{s, p}} \leq C\left(2^{j}\right)^{s-\frac{n}{p}}$.

Let $\xi=\left\{\xi_{Q}\right\}_{Q \in \mathcal{A}}$ be a countable sequence of elements from the unit ball in $\mathbf{R}^{k}$. For each $j \in \mathbf{N}$ we define

$$
f_{\xi, j}(x)=\frac{1}{j^{2}} 2^{-j \frac{\alpha}{\beta}} \sum_{Q \in \mathcal{A}_{j}} \psi_{Q}(x) \xi_{Q}
$$


and finally we set

$$
f_{\xi}=\sum_{j=1}^{\infty} f_{\xi, j}
$$

It is easy to see that $f_{\xi}$ is continuous since

$$
\left\|f_{\xi, j}\right\|_{L^{\infty}} \leq C \frac{1}{j^{2}} 2^{-j \frac{\alpha}{\beta}}
$$

By Lemma 2.2 B), \# $A_{j}=\left[2^{j \alpha}\right]$ and $\beta=\frac{p \alpha}{s p-n+\alpha}$ we obtain

$$
\left\|f_{\xi}\right\|_{W^{s, p}} \leq \sum_{j=1}^{\infty}\left\|f_{\xi, j}\right\|_{W^{s, p}} \leq C \sum_{j=1}^{\infty} \frac{1}{j^{2}} 2^{-j \frac{\alpha}{\beta}}\left(\# \mathcal{A}_{j}\right)^{\frac{1}{p}}\left(2^{j}\right)^{s-\frac{n}{p}} \leq C \sum_{j=1}^{\infty} \frac{1}{j^{2}}<\infty
$$

and hence $f_{\xi} \in W^{s, p}\left(\mathbf{R}^{n}, \mathbf{R}^{k}\right)$.

In the remaining part of the proof we would like to show that for a generic choice of $\xi$ we obtain a map $f_{\xi}$ with the desired property (5.2). Let us view $\xi=\left\{\xi_{Q}\right\}_{Q \in \mathcal{A}}$ as a sequence of independent random variables, identically distributed according to the uniform probability distribution on the unit ball $B$ in $\mathbf{R}^{k}$. Since Hausdorff and capacitary dimension coincide (see Preliminaries) it is now enough to show that for each $\beta^{\prime}<\beta(\leq k)$ we can find a measure $\mu$ on $f_{\xi}\left(M_{0} \backslash M\right)$ with finite $\beta^{\prime}$-energy.

We can fix a compact set $\tilde{M} \subset M_{0} \backslash M$ such that $0<\mathcal{H}^{\alpha}(\tilde{M})<\infty$. We consider the measure $\left(f_{\xi}\right)_{\#}\left(\left.\mathcal{H}^{\alpha}\right|_{\tilde{M}}\right)$, i.e. the pushforward of the $\mathcal{H}^{\alpha}$-measure on $\tilde{M}$ via the map $f_{\xi}$. We claim that the expectation satisfies

$$
\mathbf{E}_{\xi}\left(I_{\beta^{\prime}}\left(\left(f_{\xi}\right)_{\#}\left(\left.\mathcal{H}^{\alpha}\right|_{\tilde{M}}\right)\right)\right)<\infty
$$

for each $\beta^{\prime}<\beta$. It follows that almost surely with respect to $\xi$ we obtain that

$$
I_{\beta^{\prime}}\left(\left(f_{\xi}\right)_{\#}\left(\left.\mathcal{H}^{\alpha}\right|_{\tilde{M}}\right)\right)<\infty
$$

and our conclusion follows once we prove the claim (5.4).

Using Fubini theorem we may transform the integral from (5.4) to

$$
\int_{\tilde{M}} \int_{\tilde{M}} \mathbf{E}_{\xi}\left(\left|f_{\xi}(x)-f_{\xi}(y)\right|^{-\beta^{\prime}}\right) d \mathcal{H}^{\alpha}(x) d \mathcal{H}^{\alpha}(y)
$$

We write

$$
f_{\xi}(x)-f_{\xi}(y)=\sum_{Q \in \mathcal{A}} c_{Q}(x, y) \xi_{Q}
$$

where the coefficients for $Q \in \mathcal{A}_{j}$ are given by

$$
c_{Q}(x, y)=\frac{1}{j^{2}} 2^{-j \frac{\alpha}{\beta}}\left(\psi_{Q}(x)-\psi_{Q}(y)\right) .
$$

The sequence of the coefficients $c$ clearly belongs to $\ell^{\infty}$ and thus we may use Lemma 2.3 (note that $\beta^{\prime}<k$ ) and our task is reduced to the proof of

$$
\int_{\tilde{M}} \rho(c(x, y))^{-\beta^{\prime}} d \mathcal{H}^{\alpha}(x) \leq C<\infty
$$

where the constant $C$ is independent of $y$. Let us fix $y \in \tilde{M}$. For $x \in \tilde{M}$ let us denote by $j(x)$ the largest integer such that both $x$ and $y$ lie in the same or adjacent cubes from $\mathcal{A}_{j(x)}$. It follows that we can find $Q_{1} \ni x$ and $Q_{2} \ni y$ with $Q_{1}, Q_{2} \in \mathcal{A}_{j(x)+1}$ and $2 Q_{1} \cap Q_{2}=\emptyset$. It follows that $\psi_{Q_{1}}(x)=1$ and $\psi_{Q_{1}}(y)=0$ and hence there is a 
nonzero term in $(5.6)$ of size $\frac{1}{(j(x)+1)^{2}} 2^{-(j(x)+1) \frac{\alpha}{\beta}}$. Similar nonzero term corresponds to $\psi_{Q_{2}}$ and hence

$$
\rho(c(x, y)) \geq \frac{1}{(j(x)+1)^{2}} 2^{-(j(x)+1) \frac{\alpha}{\beta}} .
$$

For a fixed $y$ we can find at most $3^{n}$ dyadic cubes $Q \in \mathcal{A}_{j(x)}$ such that $x \in Q$ and hence the construction of $\mathcal{A}_{j}$ (5.1) gives us

$$
\mathcal{H}^{\alpha}(\{x \in \tilde{M}: j(x)=j\}) \leq 3^{n} \mathcal{H}^{\alpha}\left(Q \cap M_{0}\right) \leq C 2^{-j \alpha} .
$$

Now we can estimate

$$
\int_{\tilde{M}} \rho(c(x, y))^{-\beta^{\prime}} d \mathcal{H}^{\alpha}(x) \leq C \sum_{j=0}^{\infty} 2^{-j \alpha}\left(\frac{1}{(j+1)^{2}} 2^{-(j+1) \frac{\alpha}{\beta}}\right)^{-\beta^{\prime}} .
$$

Since $\beta^{\prime}<\beta$ it is easy to see that the series converges, which finishes our proof in the case $\alpha>n-s p$.

Now let us consider the case $\alpha>n-s p$ with $\beta=k<\frac{p \alpha}{s p-n+\alpha}$. We use the same construction but $\beta=k$ now. It is easy to check that

$$
-\frac{\alpha}{\beta}+\frac{\alpha}{p}+s-\frac{n}{p} \leq 0
$$

and hence the right hand side in (5.3) still converges and $f_{\xi} \in W^{s, p}$. The proof of $\operatorname{dim}\left(f_{\xi}\left(M_{0} \backslash M\right)\right) \geq \beta$ for a generic choise of $\xi$ remains the same.

It remains to consider the cases $\alpha \leq n-s p$ where we again use the construction with $\beta=k$. Since $\beta=k$ and $s p-n+\alpha<0$ it is easy to check (5.7) and hence $f_{\xi} \in W^{s, p}$. The proof of $\operatorname{dim}\left(f_{\xi}\left(M_{0} \backslash M\right)\right) \geq \beta$ is again the same.

Acknowledgement. The authors would like to thank Zoltán Balogh for pointing their interest to the problem.

\section{References}

[1] Adams, D. R., and L. I. HedberG: Function spaces and potential theory. - Springer-Verlag, Berlin, 1996.

[2] Astala, K.: Area distortion of quasiconformal mappings. - Acta Math. 173, 1994, 37-60.

[3] Balogh, Z. M., P. Mattila, and J. T. Tyson: Grassmannian frequency of Sobolev dimension distortion. - Comput. Methods Funct. Theory 14:2-3, 2014, 505-523.

[4] Balogh, Z. M., R. Monti, and J. T. Tyson: Frequency of Sobolev and quasiconformal dimension distortion. - J. Math. Pures Appl. 99:2, 2013, 125-149.

[5] Balogh, Z. M., J. T. Tyson, and K. Wildrick: Dimension distortion by Sobolev mappings in foliated metric spaces. - Anal. Geom. Metr. Spaces 1, 2013, 232-254.

[6] BAlogh, Z. M., J. T. TYSOn, and K. WildRICK: Frequency of Sobolev dimension distortion of horizontal subgroups of Heisenberg groups. - Preprint, 2013.

[7] Gehring, F. W.: The $L^{p}$-integrability of the partial derivatives of a quasiconformal mapping. - Acta Math. 130, 1973, 265-277.

[8] Gehring, F. W., and J. VÄısÄLÄ: Hausdorff dimension and quasiconformal mappings. - J. London Math. Soc. 6:2, 1973, 504-512.

[9] Grafakos, L.: Modern Fourier analysis. Second edition. - Grad. Texts in Math. 250, Springer, New York, 2009. 
[10] Hencl, S., and P. Honzík: Dimension of images of subspaces under Sobolev mappings. Ann. Inst. H. Poincaré Anal. Non Linéaire 29:3, 2012, 401-411.

[11] Hencl, S., and P. Honzík: Dimension of images of subspaces under mappings in TriebelLizorkin spaces. - Math. Nachr. 287:7, 2014, 748-763.

[12] Kaufman, R.: Sobolev spaces, dimension, and random series. - Proc. Amer. Math. Soc. 128:2, 2000, 427-431.

[13] Koskela, P., J. Malý, and T. ZÜrcher: Lusin's condition (N) and modulus of continuity. - Adv. Calc. Var. (to appear).

[14] Malý, J., and O. Martio: Lusin's condition (N) and mappings of the class $W^{1, n}$. - J. Reine Angew. Math. 458, 1995, 19-36.

[15] Mattila, P.: Geometry of sets and measures in Euclidean spaces. - Cambridge Stud. Adv. Math. 44, Cambridge Univ. Press, 1995.

[16] Triebel, H.: Theory of function spaces. - Monographs in Mathematics 78, Birkhäuser Verlag, Basel, 1983.

Received 6 May 2014 • Accepted 11 October 2014 